\title{
Comparison of landmark and real-time ultrasound-guided epidural catheter placement in the pediatric population: a prospective randomized comparative trial
}

\section{Tanya Mital', Manoj Kamal' ', Mritunjay Kumar' ${ }^{1}$, Rakesh Kumar' ${ }^{1}$, Pradeep Bhatia', and Geeta Singariya ${ }^{2}$}

'Department of Anesthesiology and Critical Care, All India Institute of Medical Sciences, Jodhpur, ${ }^{2}$ Dr S N Medical College, Jodhpur, India

Background: Epidural block placement in pediatric patients is technically challenging for anesthesiologists. The use of ultrasound (US) for the placement of an epidural catheter has shown promise. We compared landmark-guided and US-guided lumbar or lower thoracic epidural needle placement in pediatric patients.

Methods: This prospective, randomized, comparative trial involved children aged 1-6 years who underwent abdominal and thoracic surgeries. Forty-five children were randomly divided into two groups using a computer-generated random number table, and group allocation was performed by the sealed opaque method into either landmark-guided (group LT) or real-time ultrasound-guided (group UT) epidural placement. The primary outcome was a comparison of the procedure time (excluding US probe preparation). Secondary outcomes were the number of attempts (re-insertion of the needle), bone contacts, needle redirection, skin-to-epidural distance using the US in both groups, success rate, and complications.

Results: The median (interquartile range) time to reach epidural space was 105.5 (297.0) seconds in group LT and 143.0 (150) seconds in group UT ( $P=0.407)$. While the first attempt success rate was higher in the UT group ( $87.0 \%$ in UT vs. $40.9 \%$ in LT; P $=0.004)$, the number of bone contacts, needle redirections, and procedure-related complications were significantly lower.

Conclusions: The use of US significantly reduced needle redirection, number of attempts, bone contact, and complications. There was no statistically significant difference in the time to access the epidural space between the US and landmark technique groups.

Keywords: Acute pain; Anesthesia; Anesthesia, epidural; Pediatrics; Ultrasonography.

\section{INTRODUCTION}

Epidural analgesia (EA) is a commonly used modality for perioperative pain relief in thoracic and abdominal surgeries [1]. It provides an effective and predictable perioperative analgesia, obviates the need for systemic analgesic agents, prevents the risks of opioid-associated respiratory depression, and ensures better hemodynamic stability and recovery [2]. In children, EA through the lumbar or thoracic approach requires considerable experience and a high level of

This is an Open Access article distributed under the terms of the Creative Commons Attribution Non-Commercial License (http://creativecommons.org/licenses/by-nc/4.0) which permits unrestricted non-commercial use, distribution, and reproduction in any medium, provided the original work is properly cited.

Copyright (c) the Korean Society of Anesthesiologists, 2021 
expertise due to their small body size, narrow epidural space, and difficulty in predicting skin-to-epidural space distance. Soft connective tissue limits tactile sensation feedback for loss of resistance (LOR) in the identification of the epidural space. Moreover, these procedures are usually performed in children under deep sedation or general anesthesia (GA), and subjective warning signs (e.g., paresthesia, pain, or muscle twitch) of neural injury are missed [3]. Reports of unintentional dural puncture and spinal cord injury aggravate this fear for the safety of lumbar and thoracic epidurals in children $[4,5]$. This may also result in increased procedural time, multiple needle re-entry, and numerous bone contacts, leading to needle redirections [6].

The advent of ultrasonography has led to increased safety and success of regional anesthesia in children $[6,7]$. Due to the incomplete ossification of posterior elements of the spine in the pediatric population, an excellent acoustic window is available for visualization of structures, the needle trajectory, and its tip. The tip of the catheter, downward displacement of the dura while injection of saline or local anesthetic injection, and the spread of injected fluid can be visualized. Ultrasound (US) has been shown to improve the success rate and shorten block performance time $[3,6,7]$. US scanning can be performed before the procedure, which can help in finding the needle entry point and depth of the epidural space, but the needle is still advanced blindly.

The curiosity on using real-time US visualization for the advancement of the needle to facilitate rates of catheter placement at the lumbar spine is gradually developing $[8,9]$. Although US-guided epidural catheter insertion can be difficult in the thoracic spine, due to the narrow interlaminar space [10]. Pak and Gulati [11] performed a retrospective review of real-time thoracic epidural placement in adult patients and found it to be a successful and effective technique. They also emphasized the feasibility and the need for a prospective study comparing US-assisted epidural placement with traditional landmark-based techniques.

Due to the paucity of studies, we performed a randomized controlled trial that compared anatomical landmark-guided and the real-time US-assisted epidural catheter placement using an in-plane approach in children.

We hypothesized that a real-time in-plane ultrasound-guided technique for thoracic and lumbar epidural needle placement would be an effective technique in pediatric patients.

\section{MATERIALS AND METHODS}

This prospective, randomized, controlled trial was conducted in a tertiary care hospital after obtaining approval from the Institutional Ethical Committee and Clinical Trial Registry India (CTRI/2019/01/017022, dated 09/01/2019), between December 25, 2018, and March 20, 2020.

This clinical study was performed in accordance with the ethical principles for medical research involving human subjects outlined in the Helsinki Declaration of 1975 (revised 2013).

The study was conducted after written informed parental consent was obtained from all participants. Fifty-eight patients were enrolled in the study, of which 13 patients were excluded (not meeting the criteria $[\mathrm{n}=7]$ and declined to participate $[n=6])$. The remaining forty-five pediatric patients with the American Society of Anesthesiologists physical status I-III, aged 1-6 years, undergoing thoracic and abdominal surgeries under GA were randomized in this study (Fig. 1). Children with neurological disorders, seizures, history of spine surgeries or deformities, history of local anesthetic allergy, local site infection, or coagulopathies were excluded.

A thorough preoperative check-up of all patients was performed one day before surgery. Fasting instructions were given as per the institutional protocol. Anesthesia induction was performed using $2 \mu \mathrm{g} / \mathrm{kg}$ fentanyl and $1-2 \mathrm{mg} / \mathrm{kg}$ propofol, titrated to effect. Endotracheal intubation was facilitated by $0.5 \mathrm{mg} / \mathrm{kg}$ Atracurium. Anesthesia was maintained with sevoflurane (up to 1.5-2 vol\%), along with boluses of fentanyl and Atracurium. Intraoperative monitoring included electrocardiogram, pulse oximetry, non-invasive arterial blood pressure, esophageal temperature, capnography, and end-tidal concentrations of volatile anesthetics.

Patients were randomized into group LT (landmark technique) $(\mathrm{n}=22)$ and group UT (real-time US group) $(\mathrm{n}=23)$ by using a computer-generated random number table by an anesthesiologist who was not involved in the study. The allocated group was sealed in an opaque envelope, which was opened immediately before the procedure. In both groups, epidural placement was performed at the lower thoracic or lumbar levels in the left lateral decubitus position under all aseptic precautions.

An anesthesiologist with sufficient experience in epidural placement (at least 30 real-time US guidance or landmark guidance) in the pediatric age group conducted all of these 


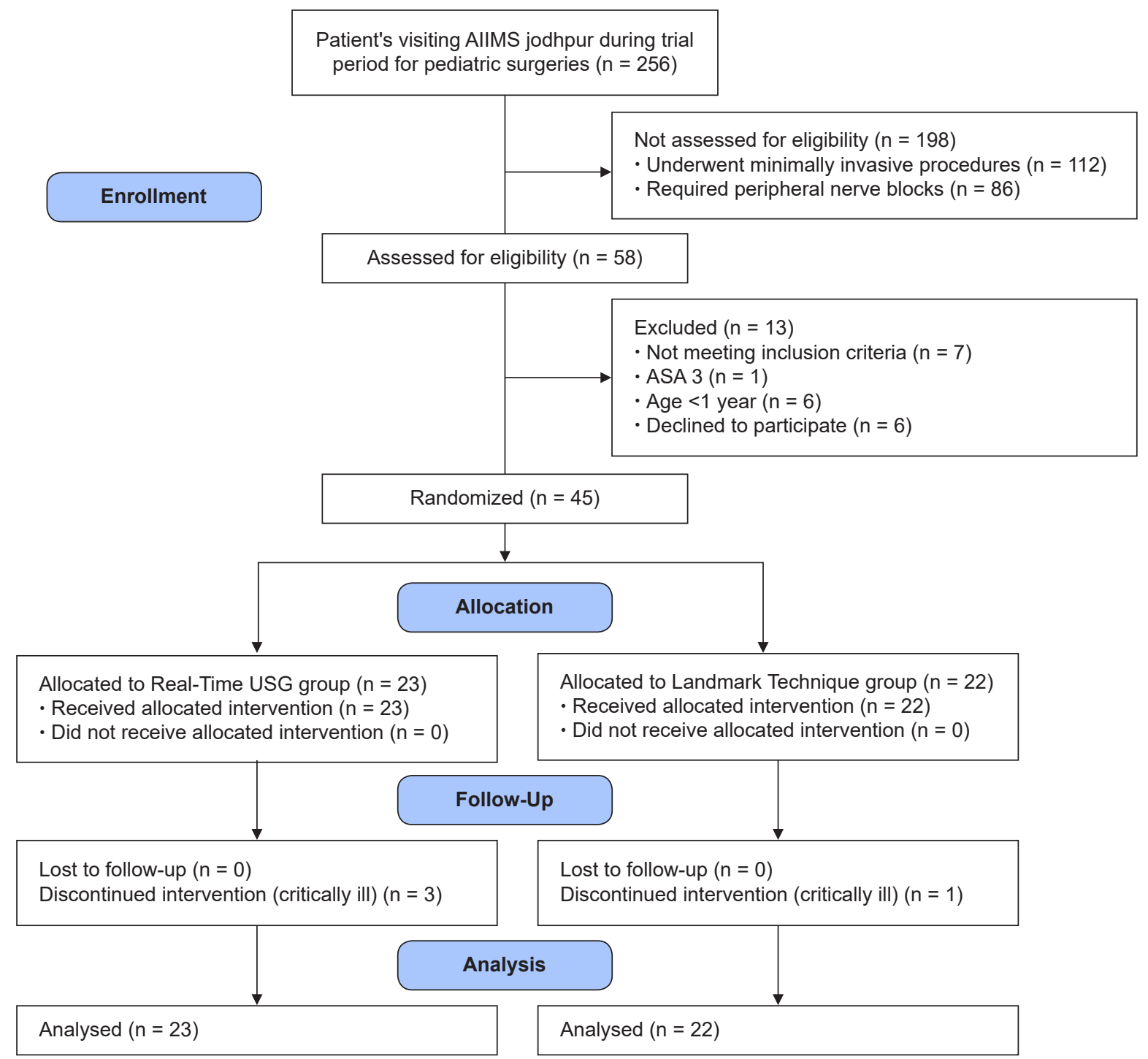

Fig. 1. Consort flow diagram. AllMS: All India Institute of Medical Sciences, Jodhpur, India, ASA: American Society of Anesthesiologists.

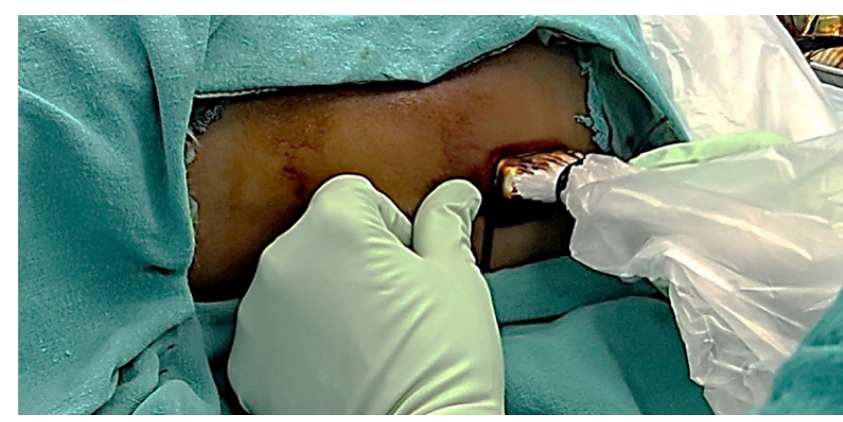

Fig. 2. Orientation of the ultrasound probe in paramedian longitudinal plane.

blocks. The imaging was performed with a hockey stick linear ultrasound probe (8-13 MHz frequency) connected to a
US machine (GE LOGIQe 9 L, GE Healthcare, USA). An epidural set containing a $20 \mathrm{G}$ Tuohy epidural needle of $50 \mathrm{~mm}$ length and a $24 \mathrm{G}$ catheter (B Braun Melsungen, Germany) was used. The level of epidural puncture was determined by the surgical incision.

In the LT group, the epidural catheter was placed under surface anatomy guidance and the standard LOR technique with air through the midline approach. The skin-to-epidural space distance was measured after the block performance using the US. The probe was applied at the congruent thoracic/lumbar level to obtain a paramedian longitudinal view of the spine (Fig. 2). The distance from the skin to the epidural space was measured in freeze mode. The spinous process was visualized as a hyperechoic structure with an 
acoustic shadow below the midline longitudinal orientation. The paramedian sagittal articular process view was then obtained by laterally moving the probe. As the articular processes were seen as a hump, the cephalad end of the probe was moved medially while maintaining a medial tilt to optimize the space between the laminae. In the paramedian sagittal oblique view, the epidural needle was inserted from the caudal end of the probe using an in-plane approach and advanced under real-time US guidance through interlaminar spaces until the tip of the needle was engaged in the ligamentum flavum. The posterior epidural space was seen as a hypoechoic area between the hyperechoic ligamentum flavum above and the shiny dura below (Fig. 3). Needle entry into the epidural space was confirmed by direct visualization of the needle tip and with the LOR to air method. We then injected saline to observe the downward displacement of the posterior dura mater (Fig. 4).

The epidural catheter was advanced $2-3 \mathrm{~cm}$ inside the epidural space and secured at the back with transparent dressings. A test dose of $0.1 \mathrm{ml} / \mathrm{kg}$ of $2 \%$ lignocaine with adrenaline $(1: 200,000)$ was administered, and the patient was placed in the supine position. The loading doses of ropivacaine $0.2 \%, 0.5 \mathrm{ml} / \mathrm{kg}$ for lumbar, and $0.3 \mathrm{ml} / \mathrm{kg}$ for thoracic epidural, respectively (total dose not exceeding 2.0 $\mathrm{mg} / \mathrm{kg}$ ), followed by $0.4 \%$ ropivacaine $(0.2 \%)$ continued for $48 \mathrm{~h}$ postoperatively.

The primary objective was to compare the procedure time by an anesthesiologist who was unaware of the technique used. The timer was started by the observer as the 20 G Tuohy needle was inserted from the caudal end of the probe and advanced under real-time US assistance with the interspace always maintained in the center of the probe. The LOR to air was used to confirm the epidural space, which marked the endpoint of the timer. The secondary objectives were the number of bone contacts, needle redirections, number of attempts (re-insertion of the needle), skin-to-epidural distance using the US in both groups, and complications such as dural puncture, failure, and a bloody tap were recorded. In the case of a dural puncture, another attempt was made at an interspace higher than the previous one. We also evaluated the ease of catheter insertion using a 3-point scale described as easy: when the catheter could be passed without any resistance, moderate: when there was some resistance to the passage, but the catheter could still be threaded, and difficult, where the catheter could not be

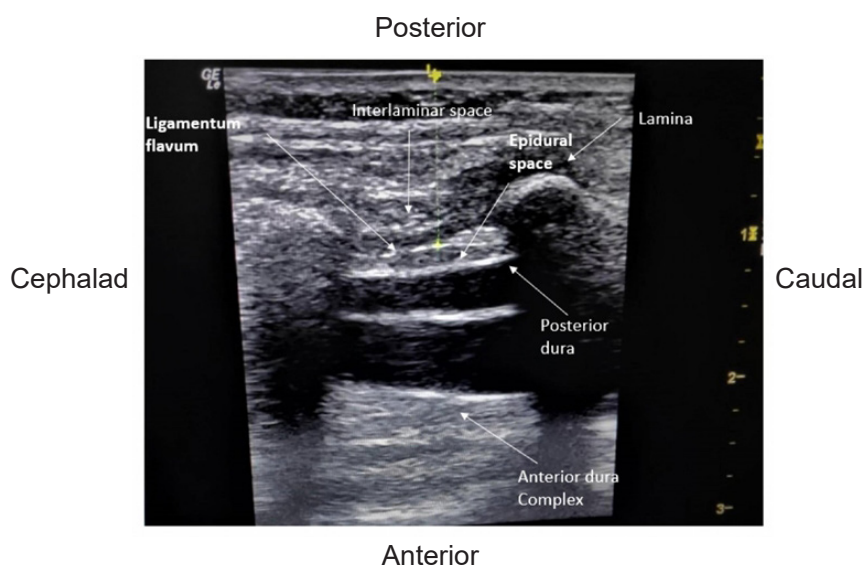

Fig. 3. Structures visualized on an ultrasound scan for epidural catheter placement.
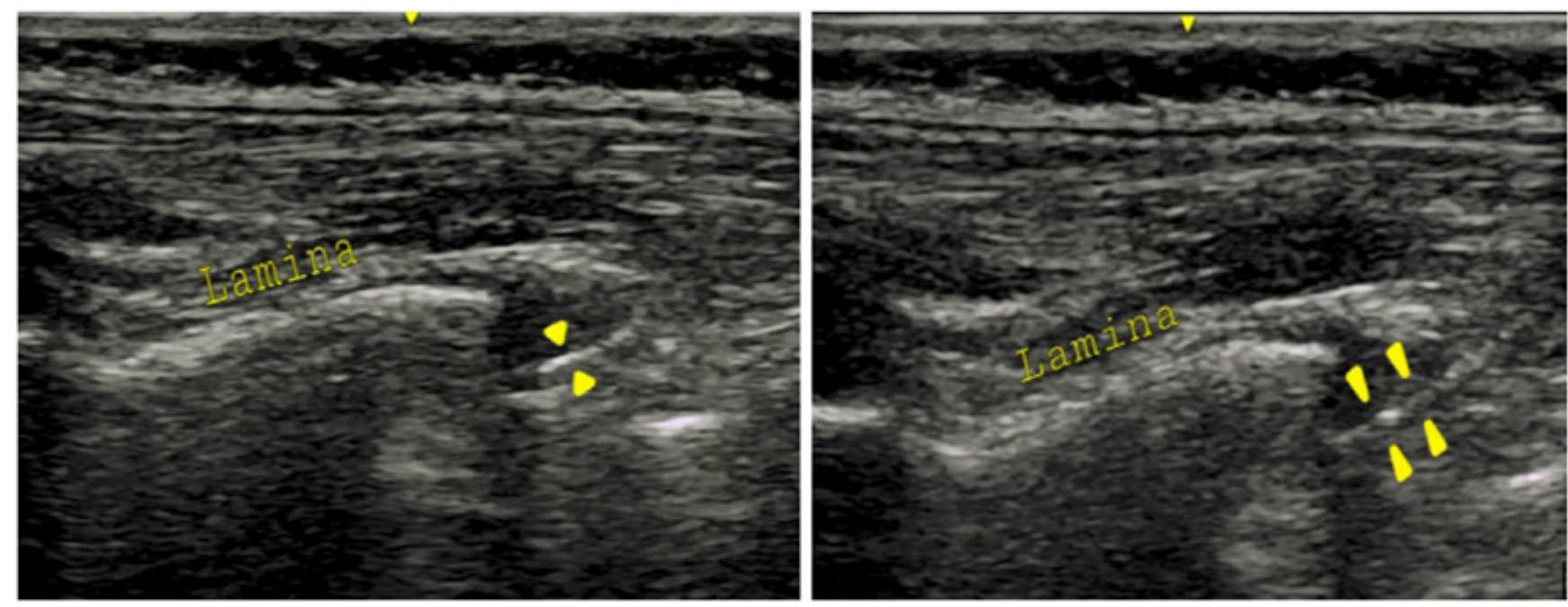

Fig. 4. Displacement of posterior dura complex on the US on saline injection. 
threaded or some manipulation of the needle was required to thread in the catheter.

The surgical incision was allowed at least $20 \mathrm{~min}$ after the epidural loading dose. When an increase in heart rate or blood pressure of more than $20 \%$ from baseline in the intraoperative or postoperative period is observed, a top-up dose of ropivacaine $0.2 \%$ and $0.25 \mathrm{ml} / \mathrm{kg}$ in cases of lumbar and $0.15 \mathrm{ml} / \mathrm{kg}$ in thoracic epidural, respectively, were administered. If hemodynamics did not revert to baseline even after the top-up dose of local anesthetic (LA), the epidural was considered as failure and fentanyl $1.0 \mu \mathrm{g} / \mathrm{kg}$ was administered. The children requiring IV analgesia during the intraoperative period were not followed for pain evaluation in the postoperative period. On completion of the surgical procedure, all children were extubated after achieving extubation criteria. Complications associated with EA such as respiratory insufficiency, bradycardia, hypotension, and hypotonia were recorded and managed accordingly.

Postoperative pain was assessed using the FLACC score at $0,6,12,24$, and $48 \mathrm{~h}$ (FLACC score $0=$ relaxed and comfortable 1-3 = mild discomfort 4-6 = moderate pain 7-10 = severe discomfort/pain). If a FLACC $\geq 4$ is noticed, a bolus dose of ropivacaine $(0.2 \%), 0.25 \mathrm{ml} / \mathrm{kg}$ of in case of lumbar $(0.15 \mathrm{ml} / \mathrm{kg})$, and the thoracic epidural was administered through the epidural catheter. Even after 20 min of a bolus injection of LA in the epidural catheter, if the FLACC is $\geq 4$, fentanyl $1.0 \mu \mathrm{g} / \mathrm{kg}$ was administered, and block failure was considered.

The sample size calculation was based on a previous study by Willschke et al. [2]. The mean time to perform epidural surgery was $142 \pm 49 \mathrm{~s}$ in the US group and $286 \pm 145 \mathrm{~s}$ in the landmark-guided technique, at a $95 \%$ confidence inter- val, an alpha error of $0.05 \%$ and $95 \%$ power, and a minimum of 30 patients were required. A total of forty-five children were enrolled in our study to cover possible dropouts.

Data were analyzed using the Statistical Package for Social Sciences (SPSS) for Windows version 23.0. (SPSS, IBM Corp, USA). The normality of the distribution was determined using the Shapiro-Wilk test. Categorical data were analyzed using the chi-square test or Fisher's exact test, as appropriate. The Mann-Whitney $U$ testwas used to compare non-normally distributed continuous data. The relationship between age, body weight, and skin-epidural depth in children was determined using the linear regression model and Pearson's correlation coefficient. Results were considered statistically significant when the $\mathrm{P}<0.05$.

\section{RESULTS}

The number of patients included in groups LT and UT was 22 and 23, respectively. Demographic data, including age, weight, and sex, were comparable between the groups. The levels of epidural placement and types of surgeries were also comparable between the two groups (Table 1). The procedural time was shorter in the group LT (105.5 [297.0] seconds; median [interquartile range]) in comparison to group UT (143.0 [150]). In $82.6 \%(n=19)$ of children, the epidural was placed in the first attempt in group UT, while it could be done only in $40.9 \%(\mathrm{n}=9)$ of children in group LT $(\mathrm{P}=0.004)$. No bone contacts were encountered in $69.6 \%(n=16)$ of the participants in group UT vs. $36.4 \%(\mathrm{n}=8)$ children in the LT group ( $\mathrm{P}=0.026)$. No needle redirections were required in $87.0 \%(n=20)$ of the UT group vs. $40.9 \%(n=9)$ in the LT group $(\mathrm{P}=0.001)$. The catheter could be easily inserted in

Table 1. Distribution of Demographic Parameters and Types of Surgery in the Two Groups

\begin{tabular}{|c|c|c|c|}
\hline Demographic parameters & Group UT $(n=23)$ & Group LT $(\mathrm{n}=22)$ & P value \\
\hline Age (mo) & $28.2 \pm 15.9$ & $36.2 \pm 20.2$ & 0.256 \\
\hline Sex & & & 0.302 \\
\hline Male & $15(65.2)$ & $11(50.0)$ & \\
\hline Female & $8(34.8)$ & $11(50.0)$ & \\
\hline Weight (kg) & $10.7 \pm 2.8$ & $11.8 \pm 3.5$ & 0.301 \\
\hline \multicolumn{4}{|l|}{ Type of surgery } \\
\hline Thoracic & $2(8.6)$ & 0 & \\
\hline Upper abdominal & $5(21.7)$ & $7(31.8)$ & \\
\hline Lower abdominal and pelvic & $16(69.5)$ & $15(68.1)$ & \\
\hline \multicolumn{4}{|l|}{ Level of epidural } \\
\hline Thoracic & $7(30.4)$ & $7(31.8)$ & \\
\hline Lumbar & $16(69.5)$ & $15(68.1)$ & \\
\hline
\end{tabular}

Values are presented as mean \pm SD or number (\%). Group LT: landmark technique group, Group UT: real-time US group. 
Table 2. Data Showing the Procedural Time, Skin-to-epidural Distance, Number of Attempts, Bone Contacts, Needle Redirections, Ease of Catheter Insertion, Complications and Postoperative IV Analgesia Requirement in the Two Groups

\begin{tabular}{|c|c|c|c|}
\hline Variables & Group UT $(n=23)$ & Group LT $(\mathrm{n}=22)$ & $P$ value \\
\hline Time taken (in seconds) & $143.0(150[25-960])$ & $105.5(297.0$ [40-650]) & 0.407 \\
\hline Skin-epidural distance (mm) & $12.9(3.3[10-17.8])$ & $15.0(5.0[10-25])$ & 0.027 \\
\hline \multicolumn{4}{|l|}{ Number of attempts } \\
\hline First attempt & $19(82.6)$ & $9(40.9)$ & 0.004 \\
\hline Two or more attempts & $4(17.4)$ & $13(59.1)$ & \\
\hline \multicolumn{4}{|l|}{ Bone contacts } \\
\hline No bone contact & $16(69.6)$ & $8(36.4)$ & 0.026 \\
\hline One or more bone contacts & $7(30.4)$ & $14(63.6)$ & \\
\hline \multicolumn{4}{|l|}{ Needle redirections } \\
\hline No needle redirections & $20(87.0)$ & $9(40.9)$ & $<0.001$ \\
\hline One or more needle redirections & $3(13.0)$ & $13(59.1)$ & \\
\hline \multicolumn{4}{|l|}{ Ease of catheter insertion } \\
\hline Easy & $20(86.9)$ & $16(72.7)$ & 0.426 \\
\hline Moderate & $2(8.6)$ & $5(22.7)$ & \\
\hline Difficult & $1(4.3)$ & $1(4.5)$ & \\
\hline \multicolumn{4}{|l|}{ Complications } \\
\hline Dura puncture & 0 & $4(18.2)$ & 0.032 \\
\hline Bloody tap & $1(4.3)$ & 0 & \\
\hline Failure & 0 & $8(36.4)$ & $<0.001$ \\
\hline Postoperative IV analgesia & 0 & $3(13.6)$ & 0.060 \\
\hline
\end{tabular}

Data are represented as median (1Q, 3Q [range]) or number (\%). Group LT: landmark technique group, Group UT: real-time US group.

$86.9 \%(n=20)$ of children in group UT and $72.7 \%(n=16)$ of children in the LT group $(\mathrm{P}=0.426)$ (Table 2$)$. The dura puncture occurred in $18.2 \%(n=4)$ of patients in the LT group, while none occurred in the UT group $(P=0.032)$. Bloody aspiration occurred in one patient in group UT. Intraoperative fentanyl was required in 8 (36.4\%) patients in the LT group, and epidural catheter placement was considered to have failed $(\mathrm{P}=0.001)$. However, none of the participants in the US group required intraoperative fentanyl supplementation (Table 2).

FLACC score $>4$ was observed in 7 children in the LT group, which was successfully treated with a bolus administration of ropivacaine. Six children in the US group also required an epidural bolus of ropivacaine in the recovery room. Three children in the LT group received postoperative fentanyl $(0.5 \mu \mathrm{g} / \mathrm{kg} \mathrm{IV})$, while none of the children in group UT required it $(\mathrm{P}=0.060)$ (Table 2$)$.

Multiple linear regression was calculated to predict the skin-epidural distance (in $\mathrm{mm}$ ) based on age in months and body weight in $\mathrm{kg}$ of children. A significant regression equation was found $(\mathrm{F}(2,42)=6.48, \mathrm{P}<0.003$, with an $\mathrm{R} 2$ of 0.23. Participants predicted that the skin-epidural distance is equal to $10.34 \pm 0.17$ (weight) +0.05 (age), where weight

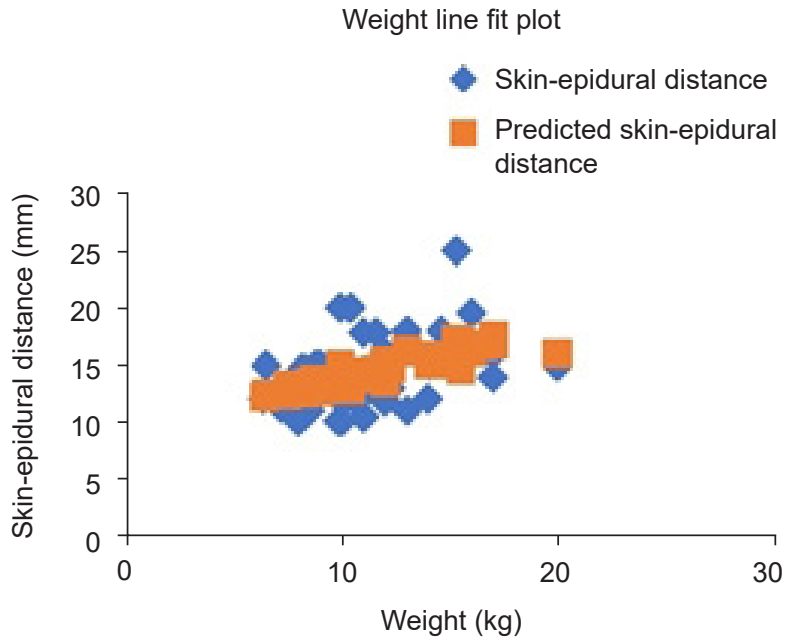

Fig. 5. Scatter plot showing the linear correlation between the body weight and the skin-epidural distance as measured on ultrasound.

is measured in kilograms and age is measured in months. The skin-epidural distance increased by $0.05 \mathrm{~mm}$ for each month of age and $0.17 \mathrm{~mm}$ for each kilogram of weight. The $\mathrm{P}$ value for age and weight as predictors of skin-epidural distance were 0.11 and 0.37 , respectively (Fig. 5). 


\section{DISCUSSION}

The results of our study showed that procedure time was shorter in group LT than in group UT, but the difference was not statistically significant. The number of bone contacts and needle redirections were fewer, and the success rate in the first attempt was higher in group UT.

Inadequate postoperative pain management in children may progress to the development of chronic pain in $20 \%$ of patients undergoing major surgery [12]. Traditionally, postoperative pain after major abdominal and pelvic pain is managed by caudal epidural block. The epidural block at the lumbar or thoracic level is technically challenging in pediatric patients because of the narrow space. The US proved a useful aid for the faster placement of the catheter along with direct visualization of the neuraxial structure and spread of LA, but there is a learning curve for this.

The procedure time in group UT was more than that in group LT, but the difference was not statistically significant. The findings of our study are in accordance with those of a study conducted by Willschke et al. [2]. In a study by Pak and Gulati [11], the mean procedure time was longer than that in our study. The longer procedure time may have been due to difficulty in discerning the ultrasonic anatomy by curvilinear, low-frequency transducers in adults.

The number of attempts, bone contacts, needle redirections, ease of catheter insertion, dural puncture, and failure rate was significantly lower in group UT than in group LT. In pediatric patients due to lack of ossification of posterior elements of the spinal canal, an acoustic window is created for the US beam to demonstrate the anatomy better. Furthermore, the shallow epidural depth in children allows a linear, high-frequency linear probe to show good image resolution and better image quality. Real-time visualization of the entire trajectory of the epidural needle during the procedure facilitates ease, accuracy, and safety [9,13-15].

A systemic review by Lam et al. [6] also demonstrated the efficacy of the US technique in terms of the reduced number of attempts, bone contacts, needle redirections, and ease of performing similar to our study. Despite the relative ease of insertion of the epidural needle under sonographic view, $30 \%$ of bone contacts were still encountered, which could be attributed to the non-visualization of the incompletely ossified bony structures, which may be missed by US imaging.

The visualization of the dura is best appreciated in younger children, and as the age advances, this visibility may gradually become difficult. The uncertainty over the depth of the epidural space has prompted few investigators to derive formulas to predict skin to the epidural distance in the pediatric population based on their age, weight, and height $[16,17]$. In our study, the correlation of skin-to-epidural distance with patient weight was also determined. The skin-to-epidural distance showed a good correlation between the age of 1-6 years and the body weight of the child.

Many methods of epidural space confirmations [18], such as LOR to air or saline or using devices like an epidural balloon, epidrum, episure, hanging drop method, epidurography, electric stimulation $[19,20]$, or electrocardiography or pressure waveform guided system [21] have been mentioned in the literature, but direct visualization of the catheter with the US and downward displacement of the dura while injecting saline or LA is the most reliable method to date.

The use of US can also mitigate complications such as dural puncture and injury to the spinal cord, encountered during traditional epidural placement in pediatric patients $[2,11,14,15]$. In our study, the success rate of epidural catheter placement was $100 \%$. Dural puncture occurred in four patients and block failure occurred in eight cases in group LT, while none occurred in group UT. Bloody tap occurred in one patient in group UT and none in group LT. This reflects an increased margin of safety for pediatric epidural placement with the use of the US. Eight children in the landmark group required additional postoperative analgesia, suggesting a higher success rate of real-time ultrasound-guided epidural insertion.

Despite the advantages of real-time US techniques, knowledge of ultrasonic anatomy and training is required, and pediatric anesthesiologists should acquire the necessary skills before actual practice.

There were some limitations to our study. The investigator was not blinded to the technique, leaving room for operator bias. The drawing conclusions on rare complications, such as a bloody tap and neural injuries, may not be accurate as they require a larger sample size.

We conclude that the use of US significantly reduced needle redirection, number of attempts, bone contact, and complications. There was no statistically significant difference in the time to access the epidural space between the US and landmark technique groups.

\section{ACKNOWLEDGEMENTS}

We acknowledge the contribution of the Department of Pediatric Surgery, All India Institute of Medical Sciences, 
Jodhpur, for helping with this study.

\section{CONFLICTS OF INTEREST}

No potential conflict of interest relevant to this article was reported.

\section{DATA AVAILABILITY STATEMENT}

The datasets generated during and/or analyzed during the current study are available from the corresponding author on reasonable request.

\section{AUTHOR CONTRIBUTIONS}

Conceptualization: Tanya Mital, Manoj Kamal, Mritunjay Kumar, Pradeep Bhatia, Geeta Singariya. Data curation: Tanya Mital, Manoj Kamal. Formal analysis: Tanya Mital, Mritunjay Kumar. Methodology: Tanya Mital, Manoj Kamal, Mritunjay Kumar, Rakesh Kumar, Pradeep Bhatia, Geeta Singariya. Project administration: Tanya Mital, Manoj Kamal, Mritunjay Kumar, Pradeep Bhatia. Writing - original draft: Tanya Mital, Manoj Kamal, Geeta Singariya. Writing review \& editing: Tanya Mital, Manoj Kamal, Mritunjay Kumar, Rakesh Kumar, Pradeep Bhatia, Geeta Singariya. Investigation: Mritunjay Kumar, Rakesh Kumar, Pradeep Bhatia, Geeta Singariya. Resources: Geeta Singariya. Supervision: Manoj Kamal, Mritunjay Kumar, Rakesh Kumar.

\section{ORCID}

Tanya Mital, https://orcid.org/0000-0002-2256-5737

Manoj Kamal, https://orcid.org/0000-0001-8314-0348

Mritunjay Kumar, https://orcid.org/0000-0001-7350-5470

Rakesh Kumar, https://orcid.org/0000-0002-4465-6138

Pradeep Bhatia, https://orcid.org/0000-0001-5082-7151

Geeta Singariya, https://orcid.org/0000-0002-1887-0851

\section{REFERENCES}

1. McNeely JK, Farber NE, Rusy LM, Hoffman GM. Epidural analgesia improves outcome following pediatric fundoplication. A retrospective analysis. Reg Anesth 1997; 22: 16-23.

2. Willschke H, Marhofer P, Bösenberg A, Johnston S, Wanzel O, Sitzwohl C, et al. Epidural catheter placement in children: comparing a novel approach using ultrasound guidance and a standard loss-of-resistance technique. Br J Anaesth 2006; 97:
200-7.

3. Kil HK. Caudal and epidural blocks in infants and small children: historical perspective and ultrasound-guided approaches. Korean J Anesthesiol 2018; 71: 430-9.

4. Rose JB. Spinal cord injury in a child after single-shot epidural anesthesia. Anesth Analg 2003; 96: 3-6.

5. Kasai T, Yaegashi K, Hirose M, Tanaka Y. Spinal cord injury in a child caused by an accidental dural puncture with a single-shot thoracic epidural needle. Anesth Analg 2003; 96: 65-7.

6. Lam DK, Corry GN, Tsui BC. Evidence for the use of ultrasound imaging in pediatric regional anesthesia: a systematic review. Reg Anesth Pain Med 2016; 41: 229-41.

7. Tsui BC, Suresh S. Ultrasound imaging for regional anesthesia in infants, children, and adolescents: a review of current literature and its application in the practice of neuraxial blocks. Anesthesiology 2010; 112: 719-28.

8. Karmakar MK, Li X, Ho AM, Kwok WH, Chui PT. Real-time ultrasound-guided paramedian epidural access: evaluation of a novel in-plane technique. Br J Anaesth 2009; 102: 845-54.

9. Grau T, Leipold RW, Fatehi S, Martin E, Motsch J. Real-time ultrasonic observation of combined spinal-epidural anaesthesia. Eur J Anaesthesiol 2004; 21: 25-31.

10. Chin KJ, Karmakar MK, Peng P. Ultrasonography of the adult thoracic and lumbar spine for central neuraxial blockade. Anesthesiology 2011; 114: 1459-85.

11. Pak DJ, Gulati A. Real-time ultrasound-assisted thoracic epidural placement: a feasibility study of a novel technique. Reg Anesth Pain Med 2018; 43: 613-5.

12. Rabbitts JA, Fisher E, Rosenbloom BN, Palermo TM. Prevalence and predictors of chronic postsurgical pain in children: a systematic review and meta-analysis. J Pain 2017; 18: 605-14.

13. Rapp HJ, Folger A, Grau T. Ultrasound-guided epidural catheter insertion in children. Anesth Analg 2005; 101: 333-9.

14. Perlas A, Chaparro LE, Chin KJ. Lumbar neuraxial ultrasound for spinal and epidural anesthesia: a systematic review and meta-analysis. Reg Anesth Pain Med 2016; 41: 251-60.

15. Guay J, Suresh S, Kopp S. The use of ultrasound guidance for perioperative neuraxial and peripheral nerve blocks in children: a cochrane review. Anesth Analg 2017; 124: 948-58.

16. Bösenberg AT, Gouws E. Skin-epidural distance in children. Anaesthesia 1995; 50: 895-7.

17. Parekh A, Dias R, Dave N. Correlation between skin-epidural space diatance with weight, age, and height in paediatric patients. Indian J Anaesth 2019; 63: 143-6.

18. Elsharkawy H, Sonny A, Chin KJ. Localization of epidural space: a review of available technologies. J Anaesthesiol Clin Pharmacol 2017; 33: 16-27. 
19. Tsui BC, Gupta S, Finucane B. Confirmation of epidural catheter placement using nerve stimulation. Can J Anaesth 1998; 45: 640-4.

20. Goobie SM, Montgomery CJ, Basu R, McFadzean J, O'Connor GJ, Poskitt K, et al. Confirmation of direct epidural catheter placement using nerve stimulation in pediatric anesthesia. Anesth Analg 2003; 97: 984-8.

21. Tsui BC. Epidural stimulation test vs epidural ECG test for checking epidural catheter placement. Br J Anaesth 2005; 95: 837. 